\title{
COMPARISON OF DIFFERENT METHODS FOR DETERMINING ATMOSPHERIC PARAMETERS OF A AND F STARS
}

\author{
B. SMALLEY \& M.M. DWORETSKY \\ Department of Physics \& Astronomy, University College London, \\ Gower Street, London WC1E 6BT, United Kingdom
}

\begin{abstract}
The atmospheric parameters of metallic-lined (Am) stars have been the subject of much debate. Using spectrophotometric flux fitting, Lane \& Lester (1984) obtained values of $\mathrm{T}_{\text {eff }}$ and $\log g$ for several classical Am stars that were systematically lower than those obtained by Dworetsky \& Moon (1986), who used empirically calibrated $u v b y \beta$ photometry. We have used the standard procedures of photometry, spectrophotometry and hydrogen-line profile fitting to determine the atmospheric parameters of many A and F stars, including several Am stars. Modifications to the standard techniques have been used to allow for the effects of cooler companion stars in the determination of such parameters. It is found that spectrophotometric methods give results for $\mathrm{T}_{\text {eff }}$ and $\log g$ that are highly sensitive to the adopted metal abundance. This explains the anomalous results of Lane \& Lester.
\end{abstract}

\section{INTRODUCTION}

Recent work on the atmospheric parameters of classical metallic-lined (Am) stars has yielded large discrepancies between the effective temperatures and surface gravities obtained from different methods. In their analysis of several Am stars Lane \& Lester (1984) obtained values for $\mathrm{T}_{\text {eff }}$ and $\log g$ that were systematically lower than those obtained previously using standard photometric methods. The use of $u v b y \beta$ photometry in the determination of such parameters was discussed by Moon \& Dworetsky (1985) and Dworetsky \& Moon (1986) who concluded that their empirically calibrated photometric grids ought to give reliable values for Am stars. A comparison between the two methods is presented below.

Recent work by Mégessier \& Van't Veer (1991) using the Infra-Red Flux Method (Blackwell \& Shallis, 1977; Blackwell, Petford \& Shallis, 1980) obtained a $T_{\text {eff }}$ for 63 Tau that was slightly lower than that obtained by Van't Veer, Cayrel \& Coupry (1991) from hydrogen-line profiles. Smalley's (1992) study of hydrogen-line profiles allows us to reconcile results for 63 Tau and other stars, from Balmer lines, uvby $\beta$ photometry, spectrophotometry and the IRFM, provided that proper allowance is made for differing $[\mathrm{M} / \mathrm{H}]$ and the presence of companion stars. 


\section{PHOTOMETRY VERSUS SPECTROPHOTOMETRY}

A detailed comparison of the use of photometry and spectrophotometry has been performed. Dworetsky \& Moon (1986) found that the differences in $\mathrm{T}_{\text {eff }}$ and $\log g$ were strongly correlated with the Strömgren metallicity index, $\delta m_{0}$. A new analysis using many more stars confirms these trends. Lester (1987) found evidence for a flux excess at $4785 \AA$ in Am stars, which was not present in normal A stars or in model fluxes. This excess was correlated with the difference in $T_{\text {eff }}$ from the two methods. However, for our much larger sample of stars this excess is not linearly correlated with $\Delta \mathrm{T}_{\text {eff }}$, indicating that the excess may not be the cause of the discrepancy. Indeed, medium-resolution spectra of the region around $4785 \AA$ show no sign of any flux excess. Smalley (1992) has shown that the excess is an artefact caused by the inclusion of excess C I opacity in the Kurucz (1979) model fluxes, based on the Kurucz \& Peytremann (1975) line list. Using new C I oscillator strengths, calculated by the Opacity Project (Seaton, 1987; Berrington et al., 1987), removed this anomaly.

The difference between the two methods is due to the effects of metal abundance on spectrophotometry. The $T_{\text {eff }}$ and $\log g$ obtained from spectrophotometric flux fitting is highly sensitive to the model metal abundance. Confirmation of this conclusion was obtained using hydrogen-line profiles.

\section{TEMPERATURES FROM HYDROGEN LINE PROFILES}

Medium-resolution $\mathrm{H} \beta$ and $\mathrm{H} \gamma$ profiles were obtained during an observing run with the Richardson Brealey Spectrograph on the $1.0 \mathrm{~m}$ Jacobus Kapteyn Telescope at the Observatorio del Roque de los Muchachos. The preliminary results were reported by Smalley \& Dworetsky (1991). A further analysis of the hydrogen-line profiles confirmed that the $T_{\text {eff }}$ obtained from them is highly reliable, once the effects of line-blanketing and rotational smearing have been allowed for. Metallicity effects on the $T_{\text {eff }}$ obtained from the profiles are relatively small ( $\sim 100 \mathrm{~K}$ for 0.5 dex change in $[\mathrm{M} / \mathrm{H}])$, compared to the high sensitivity of spectrophotometric flux fitting ( $\sim 300 \mathrm{~K}$ for 0.5 dex change in $[\mathrm{M} / \mathrm{H}])$.

The hydrogen-line profiles confirm that the $T_{\text {eff }}$ obtained from the Moon \& Dworetsky (1985) grids are very reliable and not affected by any flux excess around $4785 \AA$. A new calibration of $\beta$ indices using the JKT H $\beta$ profiles and synthetic spectra has been performed (Smalley, 1992). The new $\beta\left(\mathrm{T}_{\text {eff }}, \log g\right)$ calibration is in reasonable agreement with the Moon \& Dworetsky values.

\section{TEMPERATURES FROM INFRA-RED FLUX METHOD}

Mégessier \& Van't Veer (1991) applied the Infra-Red Flux Method to the Am star 63 Tau. Their result was confirmed during a study of several A and F stars. The IRFM gives very reliable results, but only for stars which are not binaries. However, 63 Tau is a very well-known spectroscopic binary and as such the standard IRFM cannot be used to determine the $T_{\text {eff }}$ of the primary. Cayrel, Burkhart \& Van't Veer (1991) suggested that allowing for the cool companion might raise the estimated $T_{\text {eff }}$ of the primary by $\sim 100 \mathrm{~K}$. A modified IRFM has been developed which makes allowances for the effects of the cooler companion 
(Smalley, 1992). Figure 1 shows the effect of varying the $T_{\text {eff }}$ of the secondary on the derived primary $T_{\text {eff }}$. For the expected secondary spectral type, $\mathrm{K} 5 \mathrm{~V}$ G5V (Hundt, 1972), the primary $T_{\text {eff }}$ is $\sim 7500 \mathrm{~K}$. This value agrees with that obtained from the hydrogen-line profiles.

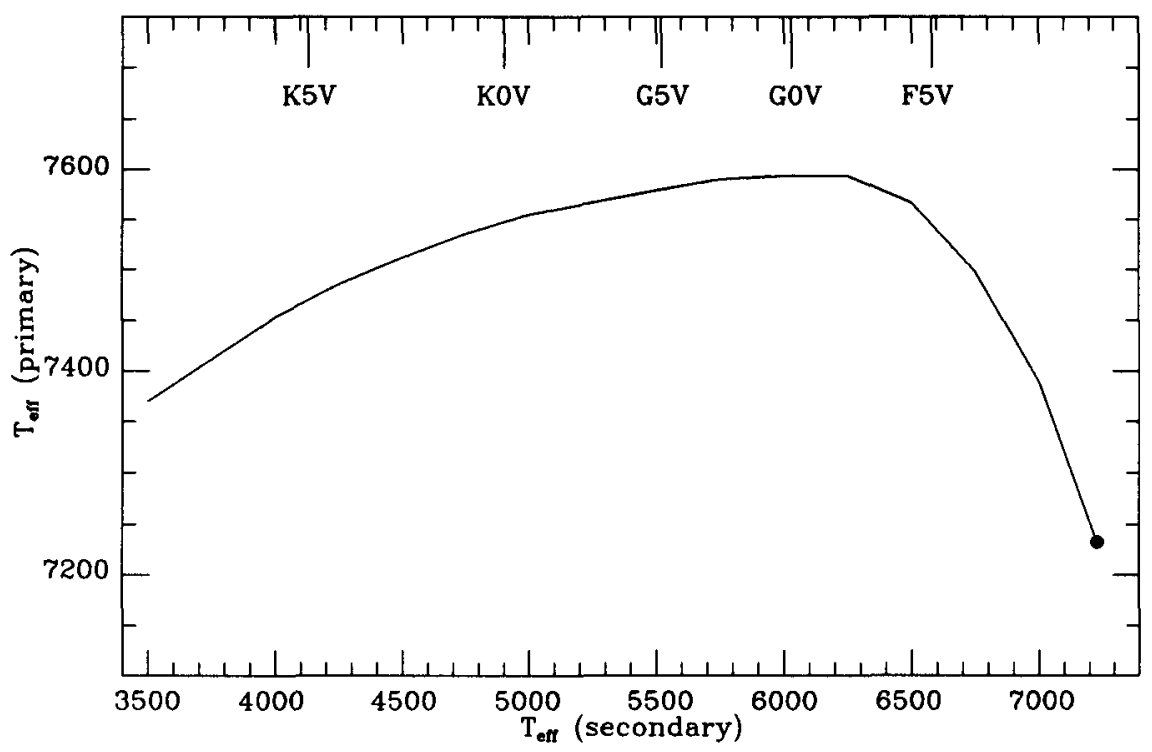

Fig. 1. The application of the Infra-Red Flux Method to a spectroscopic binary. Results for 63 Tau are given as an example of the effect of a cooler companion on the derived effective temperature of the primary. The filled circle is the $T_{\text {eff }}$ obtained by treating the binary as a single object.

\section{SURFACE GRAVITIES OF Am STARS}

The rather low $\log g$ values for Am stars obtained by Lane \& Lester (1984) implied that the Hyades Am stars were considerably evolved off the main sequence. Using the mass-luminosity relationship (Smith, 1983), it is confirmed that the Am stars in the Hyades have $\log g$ values in agreement with those obtained from $u v b y \beta$ photometry. The evolutionary tracks of Maeder \& Meynet (1988) confirm this result. An observational HR diagram shows that the Am stars in the Hyades are main-sequence objects.

\section{METAL ABUNDANCES OF Am STARS}

Using the medium-resolution spectra obtained during the observing run, a value of $[\mathrm{M} / \mathrm{H}]$ was obtained for each programme star by a comparison with synthetic spectra calculated using the Kurucz \& Peytremann (1975) and Kurucz (1988) 
line lists, for various values of $T_{\text {eff }}, \log g$ and $[\mathrm{M} / \mathrm{H}]$. Comparison of the amount of line-blocking with that predicted by the synthetic spectra for the $T_{\text {eff }}$ and $\log g$ of the star allowed the determination of $[\mathrm{M} / \mathrm{H}]$. Using these results it is found that the photometric metallicity indices are very robust stellar $[\mathrm{M} / \mathrm{H}]$ parameters of late-A and F stars. For stars in the range A3 - F0 a very tight linear correlation is obtained:

$$
[\mathrm{M} / \mathrm{H}]=-10.56 \delta m_{0}+0.081
$$

which is valid for metal-rich stars, including classical Am stars. The result is consistent with those obtained for metal-poor stars and later-type stars (Crawford, 1975; Berthet, 1990).

\section{RESULTS}

Table I gives our adopted atmospheric parameters of some well-known $\mathbf{A}$ and $\mathbf{F}$ stars, including several studied by Lane \& Lester $(1984,1987)$ and Dworetsky \& Moon (1986). The values of $\mathrm{T}_{\text {eff }}$ and $\log g$ were obtained using temperaturegravity (Kiel) diagrams and are the best estimates based on the values given by the various methods described above. Several of the stars are binaries and allowances have been made for the relative effects of the secondary companions. A much larger number of stars were analysed by Smalley (1992) and the full set of results will be published elsewhere.

TABLE I Atmospheric Parameters

\begin{tabular}{|c|c|c|c|c|c|c|}
\hline HR & star & Sp. Type. & $T_{\text {eff }}$ & $\log g$ & {$[\mathrm{M} / \mathrm{H}]$} & \\
\hline 114 & 28 And & A9IV & $7300 \pm 150$ & $3.7 \pm 0.2$ & $-0.06 \pm 0.12$ & \\
\hline 1368 & $60 \mathrm{Tau}$ & $\mathrm{kA} .3 \mathrm{hF} 2 \mathrm{mF} 2$ & $7250 \pm 100$ & $4.1 \pm 0.1$ & $0.35 \pm 0.10$ & \\
\hline 1376 & 63 Tau & $\mathrm{kA} 2 \mathrm{hF} 0 \mathrm{mF} 3$ & $7500 \pm 100$ & $4.1 \pm 0.1$ & $0.65 \pm 0.10$ & \\
\hline 1389 & 68 Tau & A2IV-Vs & $9000 \pm 200$ & $4.0 \pm 0.2$ & $0.16 \pm 0.19$ & \\
\hline 1428 & $81 \mathrm{Tau}$ & kA5hA7mF2 & $7650 \pm 100$ & $4.1 \pm 0.1$ & $0.47 \pm 0.11$ & \\
\hline 2085 & $\eta$ Lep & F1V & $7050 \pm 100$ & $4.2 \pm 0.1$ & $-0.06 \pm 0.11$ & \\
\hline 3624 & $\tau \mathrm{UMa}$ & kA3hF2mF5 & $7400 \pm 100$ & $3.9 \pm 0.1$ & $0.77 \pm 0.09$ & \\
\hline 4300 & 60 Leo & $\mathrm{A} 0.5 \mathrm{mA3V}$ & $9200 \pm 150$ & $4.1 \pm 0.2$ & $0.47 \pm 0.16$ & \\
\hline 7001 & $\alpha$ Lyr & A0Va & $9500 \pm 150$ & $3.9 \pm 0.1$ & $-0.46 \pm 0.30$ & \\
\hline 7653 & $15 \mathrm{Vul}$ & $\mathrm{kA} 5 \mathrm{hA} 7 \mathrm{~mA} 7$ & $8000 \pm 150$ & $3.7 \pm 0.2$ & $0.11 \pm 0.14$ & \\
\hline 8410 & $32 \mathrm{Aqr}$ & $\mathrm{kA} 5 \mathrm{hA} 7 \mathrm{mF} 0$ & $7900 \pm 100$ & $3.8 \pm 0.2$ & $0.46 \pm 0.11$ & \\
\hline
\end{tabular}

f Spectroscopic binary, allowance made for companion

\section{CONCLUSION}

A detailed comparison between photometry, spectrophotometry, hydrogen-lines and the Infra-Red Flux Method has been performed. The use of spectrophotometry by Lane $\&$ Lester (1984) gave values of $\mathrm{T}_{\text {eff }}$ and $\log g$ which were systematically lower than those obtained by photometry due to inadequate allowance for metal abundance. Spectrophotometric flux fitting gives values of $\mathrm{T}_{\text {eff }}$ and $\log g$ 
in agreement with $u v b y \beta$ photometry provided that the stellar metal abundance is properly allowed for in the fitting process.

The Infra-Red Flux Method yields excellent values of $T_{\text {eff }}$ and is not significantly affected by metal abundance variations. However, the method is highly sensitive to the presence of cool companion stars and hence allowance must be made for such companions, even if only approximately.

All the methods yield consistent values of $T_{\text {eff }}$ and $\log g$ once the correct metal abundance and companion $\mathrm{T}_{\text {eff }}$ and $\log g$ are used.

\section{REFERENCES}

Berrington, K.A., Burke, P.G., Butler, K., Seaton, M.J., Storey, P.J. \& Taylor, K.T., 1987. J. Phys. B: At. Mol. Phys., 20, 6379.

Berthet, S., 1990. Astr. Astrophys., 236, 440.

Blackwell, D.E. \& Shallis, M.J., 1977. Mon. Not. R. astr. Soc., 180, 177.

Blackwell, D.E., Petford, A.D. \& Shallis, M.J., 1980. Astr. Astrophys., 82, 249. Cayrel, R., Burkhart, C. \& Van't Veer, C., 1991. In: Evolution of Stars: The

Photospheric Abundance Connection, IAU Symp. No. 145, eds. Michaud,

G. \& Tutukov, A., p. 99, Kluwer, Dordrecht, Holland.

Crawford, D.L., 1975. Astr. J., 80, 955.

Dworetsky, M.M. \& Moon, T.T., 1986. Mon. Not. R. astr. Soc., 220, 787.

Hundt, E., 1972. Astr. Astrophys., 21, 413.

Kurucz, R.L., 1979. Astrophys. J. Suppl., 40, 1.

Kurucz, R.L., 1988. Private communication.

Kurucz, R.L. \& Peytremann, E., 1975. Smithsonian Ap. Obs. Spec. Rept., 362.

Lane, M.C. \& Lester, J.B., 1984. Astrophys. J., 281, 723.

Lane, M.C. \& Lester, J.B., 1987. Astrophys. J. Suppl, 65, 137.

Lester, J.B., 1987. Mon. Not. R. astr. Soc., 227, 135.

Maeder, A. \& Meynet, G., 1988. Astr. Astrophys. Suppl., 76, 411.

Mégessier, C. \& Van't Veer, C., 1991. In: Evolution of Stars: The Photospheric

Abundance Connection, Posters presented at IAU Symposium 145, eds.

Michaud, G., Tutukov, A. \& Bergevin, M., p. 35, University of Montreal.

Moon, T.T. \& Dworetsky, M.M., 1985. Mon. Not. R. astr. Soc., 217, 305.

Seaton, M.J., 1987. J. Phys. B: At. Mol. Phys., $20,6363$.

Smalley, B. PhD thesis, University of London, 1992.

Smalley, B. \& Dworetsky, M.M., 1991. In: Evolution of Stars: The Photospheric Abundance Connection, Posters presented at IAU Symposium 145, eds. Michaud, G., Tutukov, A. \& Bergevin, M., p. 47, University of Montreal. Smith, R.C., 1983. Observatory, 103, 29.

Van't Veer, C., Cayrel, R. \& Coupry, M.F., 1991. In: Evolution of Stars: The Photospheric Abundance Connection, Posters presented at IAU Symposium 145, eds. Michaud, G., Tutukov, A. \& Bergevin, M., p. 49, University of Montreal. 